\title{
Estimating affinities of calcium ions to proteins
}

This article was published in the following Dove Press journal:

Advances and Applications in Bioinformatics and Chemistry

13 March 2010

Number of times this article has been viewed

\section{Stefan Franke \\ Julia Herfurth \\ Daniel Hoffmann}

Department of Bioinformatics/Centre for Medical Biotechnology, University of Duisburg-Essen, Essen, Germany
Correspondence: Daniel Hoffmann Department of Bioinformatics/Centre for Medical Biotechnology, University of Duisburg-Essen, 451 17 Essen, Germany Tel $+49201 \quad 1834391$

Fax +49 20I 1833437

Email daniel.hoffmann@uni-due.de
Abstract: $\mathrm{Ca}^{2+}$-ions have a range of affinities to different proteins, depending on the various functions of these proteins. This makes the determination of $\mathrm{Ca}^{2+}$-protein affinities an interesting subject for functional studies. We have investigated the performance of two methods - Fold-X and AutoDock vina - in the prediction of $\mathrm{Ca}^{2+}$-protein affinities. Both methods, although based on different energy functions, showed virtually the same correlation with experimental affinities. Guided by insight from experiment, we further derived a simple linear model based on the solvent accessible surface of $\mathrm{Ca}^{2+}$ that had practically the same performance in terms of absolute errors as the more complex docking methods.

Keywords: metal ions, binding, free energy, crystal structure, solvent accessible surface

\section{Introduction}

Calcium ions (in the following termed $\mathrm{Ca}^{2+}$-ions or $\mathrm{Ca}^{2+}$ ) are important signalling agents that mediate a large number of intra- and extra-cellular processes, ${ }^{1}$ for instance blood clotting, neurotransmission, or muscle contraction. Many of these processes involve proteins that bind $\mathrm{Ca}^{2+}$ more or less transiently, and accordingly, with a wide range of $\mathrm{Ca}^{2+}$-protein affinities. ${ }^{2}$ In order to study the functional mechanisms of these proteins, it is desirable to determine these affinities. For some proteins these affinities have been determined experimentally, ${ }^{3}$ but to our experience these data are not available for most of the $\mathrm{Ca}^{2+}$-binding proteins. Given that the Protein Data Bank $\left(\mathrm{PDB}^{4}\right)$ currently contains about five thousand structures of such proteins, it would be attractive for mechanistic studies to have a method at hand to quickly estimate $\mathrm{Ca}^{2+}$-protein affinities based on the structures of the corresponding complexes.

Theoretically, it should be possible to compute the $\mathrm{Ca}^{2+}$-protein affinity by free energy techniques based on physical models, eg, by pulling the ion out of its pocket with a series of umbrella potentials in molecular dynamics simulations and integrating over the potential of mean force, ${ }^{5}$ or by the Molecular Mechanics - Poisson-Boltzmann/ Surface Area (MM-PB/SA) method. ${ }^{6}$ Despite their indisputable potential, these techniques have in the case of $\mathrm{Ca}^{2+}$-protein affinity so far not been demonstrated quantitative agreement with experiment, and, in addition, they are relatively costly in terms of computational resources. An alternative would be an estimation using empirical approaches. An early attempt towards a fast estimation of $\mathrm{Ca}^{2+}$-protein affinity was the work by Boguta et $\mathrm{al}^{7}$ who related secondary structure information with $\mathrm{Ca}^{2+}$-protein affinity. They found that for some proteins these relations could be used to classify their affinities, while for other proteins their scheme was less successful. ${ }^{8}$ More recently, submit your manuscript | www.dovepress.com

Dovepress
Advances and Applications in Bioinformatics and Chemistry 2010:2 I-6

(C) 2010 Franke et al, publisher and licensee Dove Medical Press Ltd.This is an Open Access article which permits unrestricted noncommercial use, provided the original work is properly cited. 
Schymkowitz et $\mathrm{al}^{9}$ have published Fold-X, a method and empirical force field developed for, amongst other things, the fast prediction of the binding sites and affinities of metal ions, including $\mathrm{Ca}^{2+}$ and its rival $\mathrm{Mg}^{2+}$. Since Fold-X is a kind of docking method for special ligands, it would be interesting to compare the predictive performance of this method with that of a non-specialized state-of-the-art docking method in order to assess the advancement achieved by the special parametrization of protein-metal-ion interactions in Fold-X.

The importance of $\mathrm{Ca}^{2+}$-protein binding has over the years led to large body of experimental work from which a qualitative picture has emerged of the factors that govern $\mathrm{Ca}^{2+}$-protein affinity. ${ }^{2,3}$ It is particularly notable that $\mathrm{Ca}^{2+}-$ binding usually seems to be dominated by a gain in entropy, probably due to the release of water molecules from the solvation shell of the ion; in other words: the less ligands of the protein-bound $\mathrm{Ca}^{2+}$ are water molecules (and the more are functional groups of the protein), the tighter the binding. Such qualitative models may also be helpful for guiding the development of methods for affinity estimation.

In what follows we will address some of the points raised above, namely, we will compare the correlation of experimental affinities with affinities predicted by Fold-X and the state-of-the-art docking method AutoDock vina. ${ }^{10}$ The surprising finding of this comparison has prompted further study to possibly identify more simple computational models with the same power and speed in estimation of $\mathrm{Ca}^{2+}$-protein affinities. We will show that such models can be found and that they reflect knowledge attained by experimental work.

\section{Materials and methods}

We compared two docking methods for their ability to estimate $\mathrm{Ca}^{2+}$-protein affinities, Fold- $\mathrm{X}^{9}$ and AutoDock vina. ${ }^{10}$ Fold-X has been published as a method for the prediction of positions of metal-ions on proteins, and for the prediction of affinities between proteins and metal-ions. The energetic model underlying Fold-X has an ad hoc form with a number of parameters that have been fitted to experimental data. ${ }^{9} \mathrm{We}$ obtained Fold-X versions 2.5.2 and 3.0b3 as executables for Linux from the respective server, including documentation. Despite considerable efforts we were not able to generate estimates of affinities of metal-ions to proteins using the commands described in the documentation of the software; we suspect that the option in those versions of the software is dysfunctional. Fortunately, the authors of Fold-X have offered as Table 4 in their supplementary material to Ref ${ }^{9}$ a list of $48 \mathrm{Ca}^{2+}$-binding pockets in 19 X-ray structures, mostly with experimentally determined affinities, and affinities predicted with an earlier version of Fold-X. Hence, we took these data ("Fold-X dataset") as basis for the comparison, specifically the columns "experimental energy" and "predicted energy" of Table 4 in Ref. ${ }^{9}$ For five of the binding pockets, two experimental energies were given; since the differences between the first and second energies were relatively small, only the first value was considered in each case. For ease of comparison with $\operatorname{Ref}^{9}$ we give all affinities in units of $\mathrm{kcal} / \mathrm{mol}(1 \mathrm{kcal} / \mathrm{mol}=4.1868 \mathrm{~kJ} / \mathrm{mol})$.

The 19 X-ray structures of the Fold-X dataset were retrieved from the Protein Data-bank $\left(\mathrm{PDB}^{4}\right)$ for comparative analysis with AutoDock vina. ${ }^{10}$ AutoDock vina version 1.0.3 for Linux was downloaded as executable from the website of its authors.

Usually, some information is missing from X-ray structures that is needed for energy calculations. Most importantly, this is the case for hydrogen positions, including also hydrogen bond networks. Related to this is the possibility to optimize X-ray structures by flipping carbonyl-oxygens and -NH groups (both groups have similar electron densities). Finally, sometimes X-ray structures contain atomic overlaps that can be removed relatively easily. To see whether by considering these effects, affinity predictions can be improved, we used three different protocols with AutoDock vina.

In the first protocol, PDB files were prepared with the AutoDockTool suite of AutoDock $4^{11}$ by removing water molecules, non-standard residues, and alternate positions of residues. Then polar hydrogens were added to the protein in standard orientation without rotational optimization, and Gasteiger charges ${ }^{12}$ were computed for protein atoms by AutoDock. Finally, all $\mathrm{Ca}^{2+}$-ions in the protein were redocked with AutoDock vina using default parameters, except for the "search space", ie, the volume in which the optimal docking position is searched for. Since we were interested in affinities at the crystallographically determined positions of the $\mathrm{Ca}^{2+}$-ions, and not in finding optimal positions, we restricted the search space to the minimum allowed by AutoDock vina around the crystallographically determined positions, namely a cube of $1 \AA(0.1 \mathrm{~nm})$ length in $\mathrm{x}-, \mathrm{y}-$, and $\mathrm{z}$-directions around the crystallographic positions of the $\mathrm{Ca}^{2+}$-ions. This protocol was applied to all $\mathrm{Ca}^{2+}$-ions in the Fold-X dataset.

In the second protocol we introduced in the preparation of the protein structures a further step in which the "reduce" method ${ }^{13}$ was used to optimize positions of hydrogen atoms around crystallographically determined heavy-atom positions, including also potential flips of amide-groups in side 
chains of asparagine and glutamine. Otherwise this second protocol had the same elements as the first, including also the restrained docking of $\mathrm{Ca}^{2+}$-ions with AutoDock vina.

The third protocol was similar to the first one, but additionally optimized hydrogen positions with pdb2pqr, ${ }^{14}$ including debumping to avoid steric clashes and optimization of the hydrogen bonding network.

For computations of solvent accessible surfaces (SAS) we used MSMS version 2.5.7 $7^{15}$ with standard atomic radii for protein atoms and $1 \AA$ for $\mathrm{Ca}^{2+}$. All statistical analyses were carried out with R. ${ }^{16}$

\section{Results and discussion Comparison of Fold-X and AutoDock vina}

We first analyzed the correlation of $\mathrm{Ca}^{2+}$-affinity predictions by Fold-X and AutoDock vina with experimental data in the Fold-X dataset. To this end, linear models were fitted using the least-squares algorithm. The Fold-X predictions had a value of Pearson correlation coefficient $r$ of 0.67 with a leastsquare fitted straight line $\Delta G_{\text {exp }}=3.4221 \mathrm{kcal} / \mathrm{mol}+0.5854$ $\Delta G_{\text {pred }}$ (Figure 1). The predictions by AutoDock vina (using the first protocol described in "Materials and methods") had $r=0.71$ with a best-fit line $\Delta G_{\text {exp }}=-2.393 \mathrm{kcal} / \mathrm{mol}+$ $7.309 \Delta G_{\text {pred }}$ (Fig. 2). Both above correlation coefficients

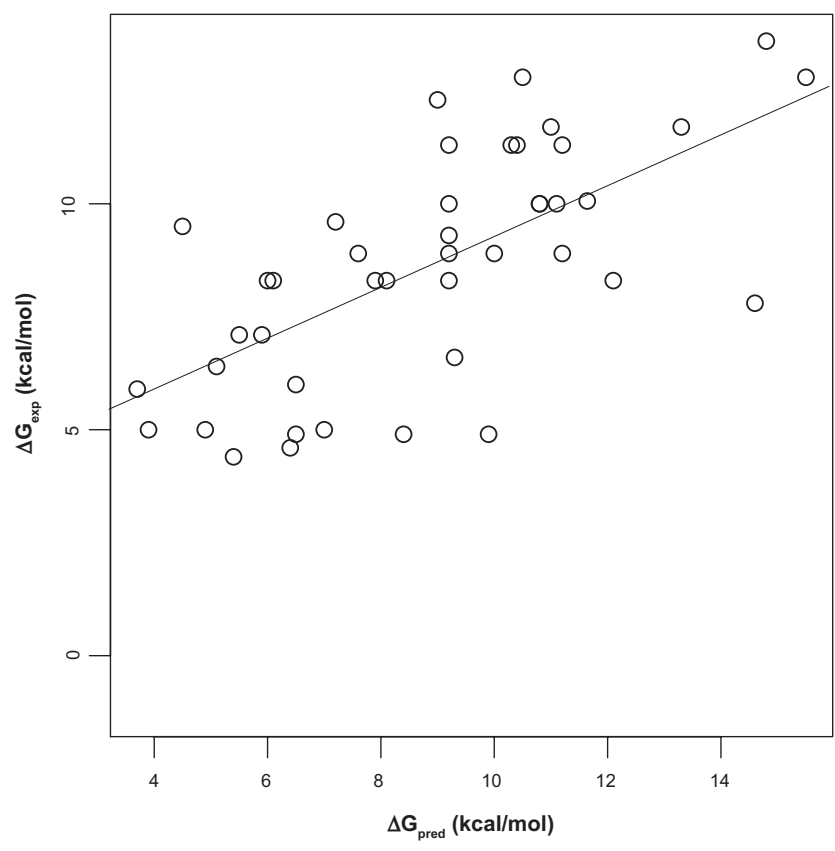

Figure I Correlation of Fold-X predictions and experiment. The straight line is a least-square fit between experimentally determined $\mathrm{Ca}^{2+}$-protein affinities $\left(\Delta G_{\text {exp }}\right)$ and affinities predicted with Fold-X $\left(\Delta G_{\text {pred }}\right)$. Pearson correlation coefficient is $r=$ 0.67. Data. ${ }^{8}$ are different from zero with high significance according to t-tests $\left(p \approx 10^{-6}\right)$. Although the range of predictions by Fold$\mathrm{X}$ has a much better overlap with the range of experimental affinities, the linear correlation as given by $r$ is slightly worse than that obtained with the AutoDock vina predictions. However, closer inspection of the data shows that the ranking of $r$-values is mainly based on a single value, the outlier in the lower left corner in Fig. 2. This outlier is given in the Fold-X dataset with a negative experimental affinity of $-1.2 \mathrm{kcal} / \mathrm{mol}$, though without reference to an experimental source of that value. The corresponding structure in the Fold-X dataset is that of a calmodulin of Paramecium tetraurelia ${ }^{17}$ (PDB entry 1 exr), and the conspicuous affinity value probably refers to an unusual fifth $\mathrm{Ca}^{2+}$-ion bound to a pocket that, according to the crystallographers, probably had been created by crystal contacts and thus may be without functional relevance. In the Fold-X dataset there is no prediction given for this pocket, while AutoDock vina produces the mentioned outlier. We can interpret this complex as representative of an extremely weakly bound $\mathrm{Ca}^{2+}$, and if we replace the experimental value of $-1.2 \mathrm{kcal} / \mathrm{mol}$ by $0 \mathrm{kcal} / \mathrm{mol}$ (ie, zero affinity) the correlation coefficient $r$ of AutoDock vina and experiment is practically unchanged at 0.71 . If we omit this experimental value altogether, the value of $r$ drops to 0.63 , which is somewhat lower than $r=0.67$ of Fold-X with experiment.

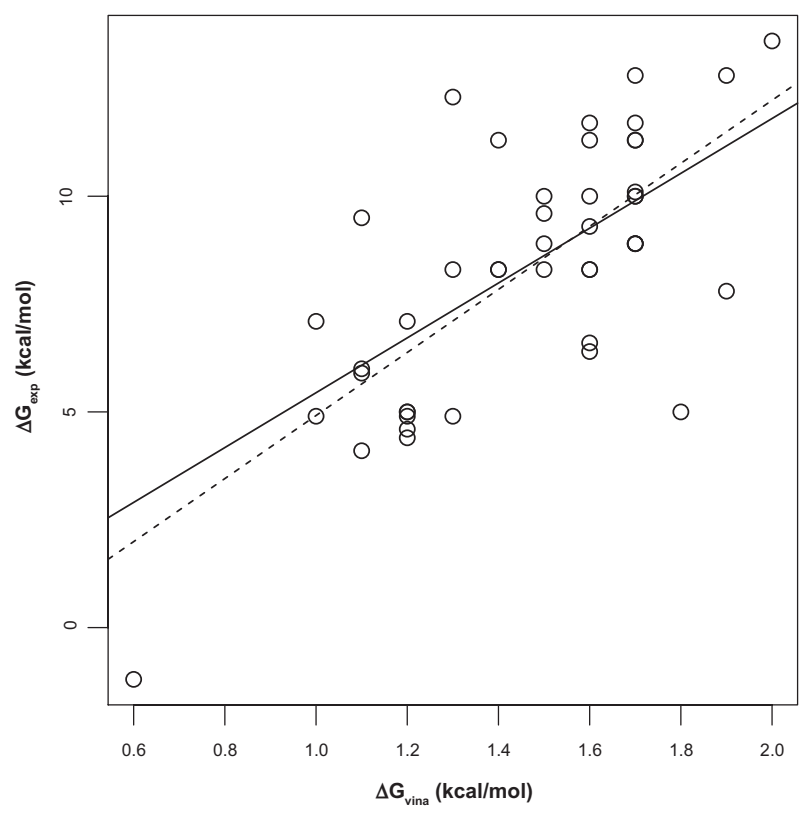

Figure 2 Correlation of AutoDock vina predictions and experiment. The dashed straight line is the least-square fit between experimentally determined $\mathrm{Ca}^{2+}$-protein affinities $\left(\Delta G_{\text {exp }}\right)$ and affinities predicted with AutoDock vina $\left(\Delta G_{\text {pred }}\right)$. Pearson correlation coefficient is $r=0.7 \mathrm{I}$. If the outlier in the lower-left is dropped, $r$ decreases to 0.63 (solid line). Experimental affinities. ${ }^{8}$ 
If we consider the $95 \%$ confidence intervals based on a t-test for $r$ we obtain [0.46, 0.81] for Fold-X vs experiment and $[0.41,0.78]$ for AutoDock vina vs experiment. Thus, the correlation coefficients between Fold-X predictions and experiment, and AutoDock vina predictions and experiment are virtually equal. This is astonishing since Fold-X had been at least partially calibrated with the same dataset, as mentioned in Ref, ${ }^{8}$ while AutoDock vina has probably not been specifically developed to solve the problem for which we have employed it here.

The two other protocols used in conjunction with AutoDock vina (see "Materials and methods") had little effect and did not improve the correlation with experiment $(r=0.71$ and $r=0.69$ for the second and third protocol, respectively). This may be due to the fact that these protocols mainly affect hydrogen positions, while $\mathrm{Ca}^{2+}$-binding pockets usually are dominated by anionic groups with few protons.

\section{A simple model for estimating $\mathrm{Ca}^{2+}$-protein affinities}

The fact that Fold-X and AutoDock vina did show the same correlation with experiment could be a consequence of the two underlying energy models capturing the same dominating cause of $\mathrm{Ca}^{2+}$-protein affinity. If this hypothesis is true, we should find a strongly decreased correlation of the two models with experiment after removing the contribution of that dominating cause out of the data.

A candidate for such a dominating effect mentioned in the introduction is the entropy gain due to water molecules that are released from the first hydration shell of $\mathrm{Ca}^{2+}$ on binding of the ion to the protein. In other words, the more water molecules are still attached to the protein-bound $\mathrm{Ca}^{2+}$, the lower the entropy gain and thus, the lower the affinity. This argument suggests an avenue to a computational test of the above hypothesis of a dominating factor: if we assume that the number of water molecules attached to the protein-bound $\mathrm{Ca}^{2+}$ is proportional to the solvent accessible surface (SAS) of the ion, we should expect a negative linear correlation of experimental free energy of binding $\Delta G_{\text {exp }}$ and SAS. The part of $\Delta G_{\text {exp }}$ not explained by the correlation with SAS is then contained in the residuals $e_{i}=\alpha S A S_{i}+\beta-\Delta G_{\text {exp }, i}$, with $\alpha$ and $\beta$ slope and intercept, respectively, of the least-squares fitted linear model, and $S A S_{i}$ and $\Delta G_{\text {exp }, i}$ the SAS and experimental affinity, respectively, of the ith $\mathrm{Ca}^{2+}$ in the Fold-X dataset. If the above hypothesis of a dominating factor is true, there should be a much smaller correlation between the predicted $\Delta G_{F o l d X, i}\left(\right.$ and $\Delta G_{\text {vina }, i}$ ) and $\mathrm{e}_{\mathrm{i}}$ as compared to the correlation between $\Delta G_{F o l d X, i}$ (and $\Delta G_{\text {vina }, i}$ ) and $\Delta G_{\text {exp }, i}$. In the following we carry out this partial correlation analysis.

As solvent probe radius we first assumed $1.4 \AA$, a value that is frequently used to model a molecular "water-sphere". With this sphere the distribution of SAS values was strongly skewed with a peak at the lowest SAS values. In fact, in thirteen of the binding pockets in the Fold-X dataset, $\mathrm{Ca}^{2+}$ was not accessible at all ( $\left.\mathrm{SAS}=0 \AA^{2}\right)$. An Anderson-Darling test rejected with high significance that the SAS values are normally distributed. Therefore, the correlation with $\Delta G_{\text {exp }}$ was not tested with Pearson correlation coefficient $r$ but with Spearman rank correlation $\rho$. We found that $\rho=-0.52$ was significantly different from zero (significance level 0.05 , $P=2 \cdot 10^{-4}$ ) (see Table 1). This correlation dropped only slightly when the outlier discussed above was omitted.

The correlation of $\Delta G_{\text {FoldX }}$ with the residuals $e_{i}$ of the least-squares fitted linear model $\Delta G_{\text {exp }}(\mathrm{SAS})$ was somewhat lower $(\rho=0.52)$ than the correlation of $\Delta G_{\text {FoldX }}$ with $\Delta G_{\text {exp }}$, but remained highly significant $\left(P=4 \cdot 10^{-4}\right)$. The same was true for $\Delta G_{\text {vina }}\left(\rho=0.50, P=4 \cdot 10^{-4}\right)$. In view of our hypothesis this means that there is an effect on the affinity that can be formulated in terms of SAS, but it may not be dominating affinity.

Our argument has so far neglected the fact that the crystal structures are results of an averaging process. A protein at ambient temperatures explores many conformations, so that the solvent accessibility of $\mathrm{Ca}^{2+}$-ions computed for the

Table I Spearman rank correlation $\rho$ of four models with experimental affinities $\Delta G_{\text {exp }}$ and residuals e

\begin{tabular}{|c|c|c|c|}
\hline Model $^{a}$ & $\rho\left(\Delta G_{\text {exp }}, \text { Model }\right)^{b}$ & $\rho\left(e_{\Delta \exp { }^{\prime} \text { SASI.4}}, \text { Model }\right)^{c}$ & $\rho\left(\mathrm{e}_{\triangle G_{\text {exp }} \text { 'SAS0.5}}, \text { Model }\right)^{\mathrm{c}}$ \\
\hline $\mathrm{SAS}_{1.4}$ & $-0.52\left(2 \cdot 10^{-4}\right)$ & & \\
\hline $\mathrm{SAS}_{0.5}$ & $-0.52\left(2 \cdot 10^{-4}\right)$ & & \\
\hline$\Delta G_{\text {Foldx }}$ & $0.66\left(2 \cdot 10^{-6}\right)$ & $0.52\left(4 \cdot 10^{-4}\right)$ & $0.39(0.01)$ \\
\hline$\Delta G_{\text {vina }}$ & $0.64\left(3 \cdot 10^{-6}\right)$ & $0.50\left(4 \cdot 10^{-4}\right)$ & $0.34(0.02)$ \\
\hline
\end{tabular}

Notes: afour linear least squares fit models with input variables SAS with probe radius I.4 A, SAS with probe radius of $0.5 \AA$ (see Eq. (I)), affinities computed with Fold-X, affinities computed with AutoDock vina; ${ }^{b}$ Spearman rank correlation $\rho$ of experimental affinities $\Delta G_{\exp }$ with predictions of fitted models; ${ }^{c} \rho$ of residuals of model based on fit with $S A S_{1.4}$ with Fold-X and AutoDock vina, respectively; ${ }^{\alpha} \rho$ of residuals of model based on fit with $S A S_{0.5}$ with Fold-X and AutoDock vina. Numbers in parentheses are $p$-values for the null-hypothesis that $\rho=0$. 
crystal structure may not reflect its true accessibility. Since $\mathrm{Ca}^{2+}$-ions in proteins are often surrounded by a tightly packed first co-ordination shell and thus have minimum solvent accessibility, we expect that conformational flexibility could perturb that packing and thus lead on average to a higher solvent accessibility. An approach that takes mobility into account could be to simulate the molecular dynamics and compute the accessibility as thermodynamic average. As this is computationally expensive, and we were more interested in a fast approximation, we tried to find a faster alternative that works in a similar direction. In a sense, the higher accessibility due to the protein flexibility can be mimicked by using a probe with a smaller radius. We therefore carried out the partial correlation analysis described above with a series of smaller probe radii between $1.4 \AA$ and $0.3 \AA$. The highest correlations of SAS with $\Delta G_{\text {exp }}$ were obtained with $0.4 \AA$ and $0.5 \AA$. As the numerically safe minimum probe radius in MSMS (see "Materials and methods") is $0.5 \AA$, we completed our analysis with this value (see Table 1 and Figure 3).

While the correlation $\rho$ of SAS with $\Delta G_{\text {exp }}$ changed only marginally from -0.52 to -0.53 and the $p$-value remained constant, the correlation of the residuals with $\Delta G_{F o l d X}$ and $\Delta G_{\text {vina }}$, respectively, dropped more strongly to 0.39 and 0.34 with $p$-values of 0.01 and 0.02 indicating no longer highly significant correlation. Omission of the discussed outlier does

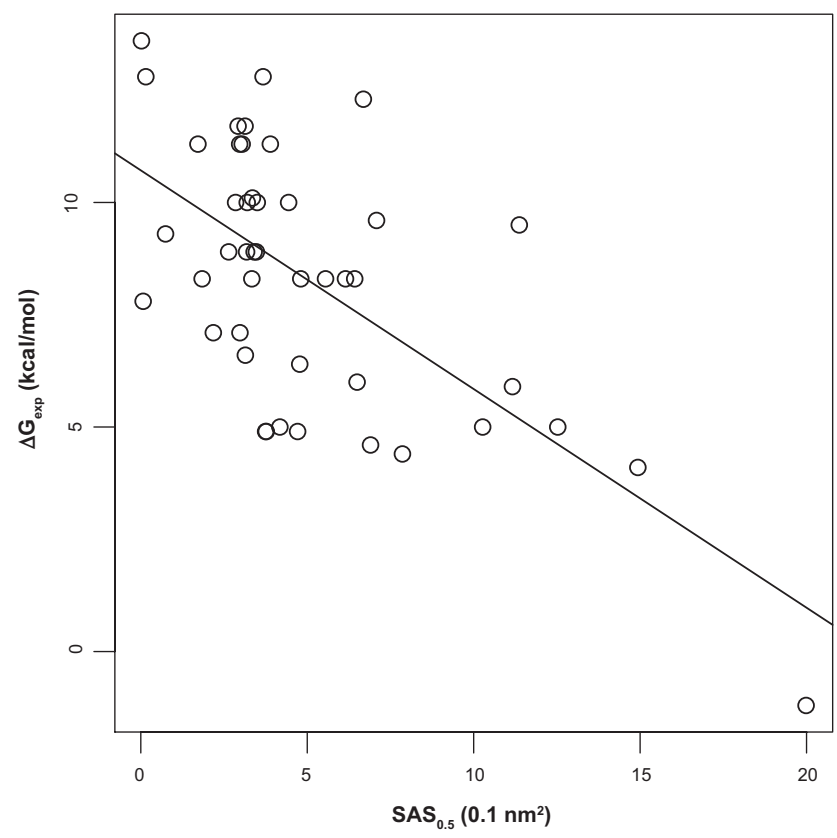

Figure 3 Correlation of SAS (probe radius $0.5 \AA$ ) and experiment. The straight line is the least-squares fit between experimentally determined $\mathrm{Ca}^{2+}$-protein affinities $\left(\Delta G_{\text {exp }}\right)$ and the solvent accessible surfaces (SAS) of the $\mathrm{Ca}^{2+}$-ions. Spearman rank correlation coefficient $\rho$ is $-0.53\left(P=2 \cdot 10^{-4}\right.$ for null-hypothesis $\left.\rho=0\right)$. Experimental affinities. ${ }^{8}$ not change the picture; conversely, one could argue that a fully solvent exposed $\mathrm{Ca}^{2+}$ with $\Delta G=0$ (which is approximately the case for the outlier) should be included in the data to represent the limiting case of no binding. Overall we can conclude that SAS with probe radius of $0.5 \AA\left(S A S_{0.5}\right)$ indeed models a dominating effect on affinity.

For a simple model to predict $\Delta G$ from SAS we did a least-squares fit of $S A S_{0.5}$ and $\Delta G_{\text {exp }}$ and found

$$
\Delta G_{\text {exp }} \approx-0.63 \cdot S A S_{0.5}+11.95
$$

with $S A S_{0.5}$ in $\AA^{2}$ and $\Delta G_{\text {exp }}$ in $\mathrm{kcal} / \mathrm{mol}$. Following the above argument we replaced in the fit the outlier by the point given by theoretical values for an unbound $\mathrm{Ca}^{2+}$, ie, $S A S_{0.5}=4 \pi\left(r_{\text {ca-ion }}+\right.$ $\left.r_{\text {probe }}\right)^{2}=4 \pi 1.5^{2}=18.85$ and $\Delta G=0$, and enforced inclusion of this point $(18.85,0)$ in the linear model. The mean of the absolute errors of this model on the Fold-X dataset was 1.9 $\mathrm{kcal} / \mathrm{mol}$, which is not much larger than the mean absolute error of $1.8 \mathrm{kcal} / \mathrm{mol}$ of Fold-X itself against $\Delta G_{\text {exp }}$.

We assessed the robustness of the model of Eq. (1) in a leave-one-out test: Each of the $\Delta G_{\text {exp }}$ values was left out of the fitting procedure, and then a model was derived from the other values. The $\Delta G_{\text {exp }}$ of the $\mathrm{Ca}^{2+}$-ion left out was then predicted by applying the new model to the $S A S_{0.5}$ value of the left-out $\mathrm{Ca}^{2+}$-ion. This was iterated over all $\Delta G$ values. The resulting mean of absolute errors was $2.0 \mathrm{kcal} / \mathrm{mol}$.

Thus, we have achieved our goal of a simple and fast computational procedure that allows an estimation of $\mathrm{Ca}^{2+}$ protein affinities based on the structure of the complexes. Judged from the numerical experiments described above, the accuracy of the method should be high enough to classify $\mathrm{Ca}^{2+}$-binding pockets into weakly or strongly binding. The accuracy is limited by several factors, of which we mention two: First, as pointed out in $\operatorname{Ref}^{8}$ the experimental data on which the model has been based may in part not satisfy modern standards. Second, the simple model of Eq. (1) does completely neglect that the binding of calcium is often accompanied by global re-arrangements of protein conformation that also affect the free energy of binding. ${ }^{2}$

Finally, we can speculate how Fold-X and AutoDock vina with their different energy functions could nevertheless capture the effect expressed in terms of SAS that we mainly attribute to the entropy gain due to release of water molecules bound to the solvated $\mathrm{Ca}^{2+}$. Neither the energy function of Fold-X nor that of AutoDock vina contains a term that explicitly takes into account this physical effect. However, both Fold-X and AutoDock vina evaluate the $\mathrm{Ca}^{2+}$-protein affinity essentially by estimating interactions of $\mathrm{Ca}^{2+}$ with the atoms of the protein lining the binding pocket. According 
to our experience these pockets are dominated by anionic groups and groups with negative partial charges. The direct interaction of $\mathrm{Ca}^{2+}$ with such negative groups is in fact taken into account by Fold-X and AutoDock vina, and this may be the cause of the apparent correlation with SAS: the more negative groups are around a $\mathrm{Ca}^{2+}$, the lower the predicted affinity due to direct interaction, but also the lower the SAS of that $\mathrm{Ca}^{2+}$, because each of the neighboring groups will supplant water molecules and lead to their release.

\section{Acknowledgments/disclosures}

The authors report no conflicts of interest in this work. Funding by BMBF grant number 01EZ0933 is gratefully acknowledged.

\section{References}

1. Berridge MJ, Bootman MD, Roderick HL. Calcium signalling: dynamics, homeosta-sis and remodelling. Nat Rev Mol Cell Biol. 2003;4:517-529.

2. Gifford JL, Walsh MP, Vogel HJ. Structures and metal-ion-binding properties of the $\mathrm{Ca}^{2+}$-binding helix-loop-helix ef-hand motifs. Biochem J. 2007;405:199-221.

3. Linse S, Forsén S. Determinants that govern high-affinity calcium binding. Adv Second Messenger Phosphoprotein Res. 1995;30:89-151.

4. Berman HM, Westbrook J, Feng Z, et al. The Protein Data Bank. Nucleic Acids Res. 2000;28:235-242.

5. Kobayashi C, Takada S. Protein grabs a ligand by extending anchor residues: molecular simulation for $\mathrm{Ca}^{2+}$ binding to calmodulin loop. Biophys J. 2006;90:3043-3051.

6. Zhao J, Nelson DJ, Huo S. Potential influence of asp in the $\mathrm{Ca}^{2+}$ coordination position 5 of parvalbumin on the calcium-binding affinity: a computational study. J Inorg Biochem. 2006;100:1879-1887.
7. Boguta G, Stepkowski D, Bierzyński A. Theoretical estimation of the calcium-binding constants for proteins from the troponin c superfamily based on a secondary structure prediction method. i. estimation procedure. J Theor Biol. 1988;135:41-61.

8. Boguta G, Stepkowski D, Bierzyński A. Theoretical estimation of the calcium-binding constants for proteins from the troponin c superfamily based on a secondary structure prediction method. ii. applications. J Theor Biol. 1988;135:63-73.

9. Schymkowitz JW, Rousseau F, Martins IC, Ferkinghoff-Borg J, Stricher F, Serrano L. Prediction of water and metal binding sites and their affinities by using the fold-x force field. Proc Natl Acad Sci U SA. 2005;102:10147-10152.

10. Trott O, Olson AJ. Autodock vina: Improving the speed and accuracy of docking with a new scoring function, efficient optimization, and multithreading. J Comput Chem. 2009;31:455-461.

11. Morris GM, Huey R, Lindstrom W, et al. Autodock4 and autodocktools4: Automated docking with selective receptor flexibility. J Comput Chem. 2009;30:2785-2791.

12. Gasteiger J, Marsili M. Iterative partial equalization of orbital electronegativity - rapid access to atomic charges. Tetrahedron. 1980;36: 3219-3228.

13. Word JM, Lovell SC, Richardson JS, Richardson DC. Asparagine and glutamine: using hydrogen atom contacts in the choice of side-chain amide orientation. J Mol Biol. 1999;285:1735-1747.

14. Dolinsky TJ, Nielsen JE, McCammon JA, Baker NA. Pdb2pqr: an automated pipeline for the setup of poisson-boltzmann electrostatics calculations. Nucleic Acids Res. 2004;32:W665-W667.

15. Sanner MF, Olson AJ, Spehner JC. Reduced surface: an efficient way to compute molecular surfaces. Biopolymers. 1996;38:305-320.

16. R Development Core Team. R: A Language and Environment for Statistical Computing. R Foundation for Statistical Computing, Vienna, Austria, 2006. ISBN 3-900051-07-0, http://www.R-project.org.

17. Wilson MA, Brunger AT. The 1.0 a crystal structure of ca(2+)-bound calmodulin: an analysis of disorder and implications for functionally relevant plasticity. J Mol Biol. 2000;301:1237-1256.

\section{Publish your work in this journal}

Advances and Applications in Bioinformatics and Chemistry is an international, peer-reviewed open-access journal that publishes articles in the following fields: Computational biomodelling; Bioinformatics; Computational genomics; Molecular modelling; Protein structure modelling and structural genomics; Systems Biology; Computational
Biochemistry; Computational Biophysics; Chemoinformatics and Drug Design; In silico ADME/Tox prediction. The manuscript management system is completely online and includes a very quick and fair peerreview system, which is all easy to use. Visit http://www.dovepress.com/ testimonials.php to read real quotes from published authors. 